Journal of Animal and Veterinary Advances 11 (1): 83-87, 2012

ISSN: $1680-5593$

(C) Medwell Journals, 2012

\title{
Mutagenicity of Sediment Samples from Nif Brook (Western Turkey)
}

\author{
${ }^{1}$ Meltem Boyacioglu, ${ }^{1}$ Hatice Parlak, ${ }^{1}$ Ozlem Cakal Arslan, \\ ${ }^{2}$ Selma Katalay and ${ }^{1}$ Muhammet Ali Karaslan \\ ${ }^{1}$ Faculty of Fisheries, Ege University, 35100 Bornova, Izmir, Turkey \\ ${ }^{2}$ Department of Biology, Faculty of Arts and Sciences, \\ Celal Bayar University, Manisa, Turkey
}

\begin{abstract}
The present study aimed to assess mutagenicity of the sediment samples from Nif brook using solvent extraction method and to compare its results with those of a previous study on mutagenicity using Soxhlet-extraction method. Sediment samples were collected from 5 sites located in Nif brook in April, 2007. Sediment samples from 5 sites on Nif brook were studied for their mutagenic potential on TA 98 and 100 strains of Salmonella typhimurium using Ames test (Plate incorporation assay) without metabolic activation. Extracts of the sediment samples were assayed in three different concentrations (In 25, 50, $100 \mu \mathrm{g}$ ). Sediment sample from Nif 5 sides was observed to be weak-mutagenic and those from Nif 1 and 4 sides were observed to have toxic effects. Toxic effects of the sediment samples on bacteria were observed on the basis of significantly reduced numbers of revertants compared to the solvent controls. According to Ames criteria, sediment samples from the Nif 5 were considered as mutagenic on TA 98 strain. According to the present study, mutagenicity was detected in Nif brook by solvent extraction method.
\end{abstract}

Key words: Ames test, Salmonella typhimurim, mutagenicity, Nif brook, sediment, Turkey

\section{INTRODUCTION}

Nif brook is located close to Kemalpasa in Izmir city on Aegean coastline of Turkey and has been polluted intensively as a consequence of big increases in population as well as numerous industrial facilities and industrial investments. Currently, there are 138 industrial facilities in the region involved in such industrial fields as food, machines, rubbers, plastics and other petro-chemical products, chemicals, textiles, electric devices, households, beverages, leather and packing materials. Of these industrial facilities, only 38 have treatment plants and water from them admixes with that of Nif brook.

Such streams as Nif brook passing through urban areas receive chemical discharges from industrial, municipal and agricultural sources. The water of Nif brook is used for domestic and industrial water supply and for purpose of irrigation. Discharge of industrial and municipal wastes to aquatic environments has contaminated sediments with a diverse variety of toxic and mutagenic chemicals.

Most of the discharged chemicals are hydrophobic in nature and sequestered by sediment particles that eventually settle in sediments (Furlong et al., 1988). They may resist microbial degradation and tend to accumulate in high concentrations in sediments or biota (Furlong et al., 1988; Alexander, 1981).
Detecting the mutagens in environmental samples is especially important because of their ability to induce cancer and potential to damage the germ line which may lead to fertility problems and potentially harmful genetic alterations in future generations (Czyz et al., 2002; Kataoka et al., 2000).

The Salmonella/microsome mutagenicity assay also called the Ames test has been widely used to detect mutagenic potential of various chemicals (Ames et al., 1975).

Using chemical tecniques however, it is not possible to determine whether a given substance has carcinogenic or mutagenic properties. Therefore, biological testing and monitoring of carcinogenic and mutagenic compounds is of particular importance (Shugart, 1995). Besides, short-term bioassays such as the Ames test have been accepted as useful in mass screening of potentially carcinogenic compounds in environmental samples (Schuetzle and Lewtas, 1986). They used the Ames test to screen sediment samples from Nif brook for their mutagenic potential as a measure of genotoxicity.

\section{MATERIALS AND METHODS}

The sediment samples were taken from several stations on Nif brook before and after it entered the

Corresponding Author: Meltem Boyacioglu, Department of Hydrobiology, Faculty of Fisheries, Ege University, 35100 Bornova, Izmir, Turkey 


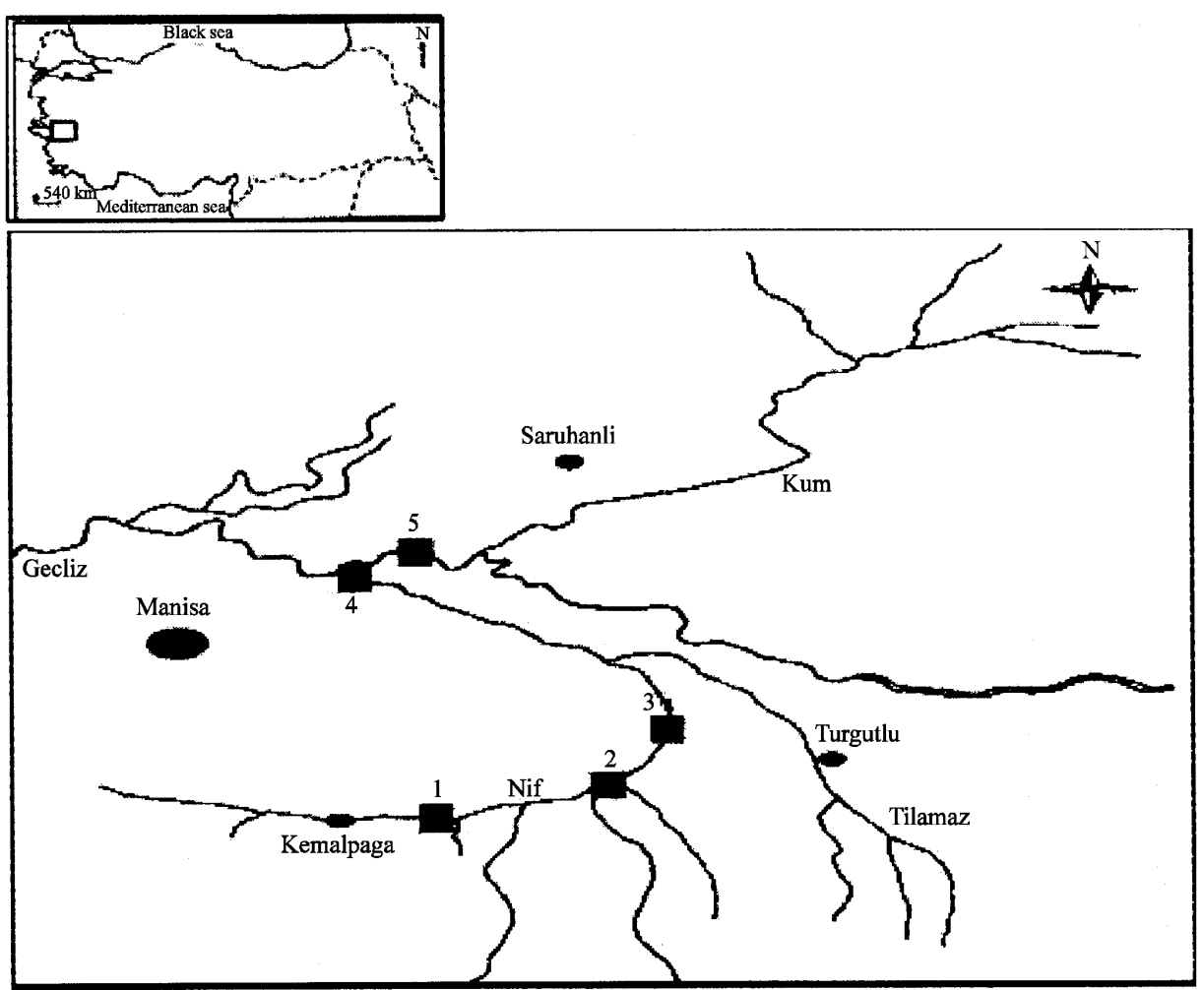

Fig. 1: Sampling sites in the study area of Nif brook

Organized Industrial Site (OIS) (Fig. 1). Sediment samples were collected by a grab sampler from 5 sides in Nif brook. The samples were stored at $4{ }^{\circ} \mathrm{C}$, prior to extraction within 1 week after collection (Maccubin et al., 1991).

Sediment samples were collected from 5 sites located in Nif brook in April, 2007 (Fig. 1). Attention was paid to select the sites located around Organized Industrial Site (OIS). The sediment samples were collected by grab sampler and placed jars and kept in ice-box until it was transferred to the laboratory. Samples were stored under cold-chain conditions and then allowed to dry at room temperature.

Sediment samples from Nif brook were crash to powder and sifted in the laboratory and then placed in portions of $1 \mathrm{~g}$ into sterile Teflon tubes and mixed with hexane/chloroform/acetone (1:1:1: v:v:v) using a vortex mixer. Subsequently, the samples were centrifuged for $10 \mathrm{~min}$ at $4^{\circ} \mathrm{C}$ at $5600 \mathrm{~g}$ in Sigma K3 cooled centrifuge and supernatants were taken into sterile tubes.

Organic solvents of the sediment samples were evaporated after this procedure had been repated for 3 times and after determining the residue content, solvent was evaporated and Dimethylsulfoxide (DMSO) was added to give a final residue concentration of $1 \mathrm{~g} \mathrm{~mL}^{-1}$ (Kotelevtsev and Stepanova, 1995).
Salmonella mutagenicity tests were performed using the standard plate incorporation method (Maron and Ames, 1983) with the TA98 and 100 strains of Salmonella typhimurium and without S9-derived metabolic activation.

Prior to Ames test, organic extracts were diluted to test 25,50 and $100 \mu \mathrm{L}$ of residue for each sample. Each of the organic extracts was tested for mutagenicity without metabolic activation using the standard plate incorporation protocol (Maron and Ames, 1983). When testing for mutagenicity without metabolic activation, $100 \mu \mathrm{L}$ of organic extract (Sediment) was mixed with $100 \mu \mathrm{L}$ of an overnight culture of bacteria and $200 \mu \mathrm{L}$ of melted agar containing $0.5 \mathrm{mM}$ histidine and biotin. The molten top agar was then poured onto a minimal glucose agar base plate and incubated at $37^{\circ} \mathrm{C}$ for 2 days. $\mathrm{NaN}_{3}\left(1.5 \mu \mathrm{g}_{\text {plate }}{ }^{-1}\right)$ and mitomycin-C $\left(0.5 \mu \mathrm{g}\right.$ plate $\left.^{-1}\right)$ were used as positive controls. Each dilution of sediment extracts and controls was assayed in triplicate. Following incubation, the number of revertant colonies (His ${ }^{-}$revertants) was counted (Maron and Ames, 1983).

\section{RESULTS AND DISCUSSION}

After 5 sediment samples were extracted, they were dissolved in DMSO and tested directly with Ames test for 
Table 1: Mutagenicity analysis of sediment samples using $S$. typhimurium assay with TA 98 strain in the absence of metabolic activation

\begin{tabular}{lccclc}
\hline TA 98 & \multicolumn{1}{c}{$\mathrm{NC}^{\mathrm{a}}$} & \multicolumn{1}{c}{$25 \mu \mathrm{g}$} & \multicolumn{1}{c}{$50 \mu \mathrm{g}$} & $100 \mu \mathrm{g}$ & $\mathrm{A}-\mathrm{Cr}$ \\
\hline Nif 1 & $28.0 \pm 5.0$ & $34.6 \pm 2.00^{*}$ & $26.6 \pm 4.5$ & $31.6 \pm 2.3$ & $\mathrm{NM}$ \\
Nif 2 & $17.3 \pm 3.7$ & $23.6 \pm 9.00^{*}$ & $20.6 \pm 3.5$ & $20.6 \pm 2.5^{*}$ & $\mathrm{NM}$ \\
Nif 3 & $17.3 \pm 3.7$ & $20.6 \pm 3.70$ & $18.6 \pm 0.5$ & $22.0 \pm 4.5^{*}$ & NM \\
Nif 4 & $26.6 \pm 3.2$ & $29.6 \pm 3.50$ & $27.0 \pm 2.6$ & $31.0 \pm 3.0$ & NM \\
Nif 5 & $17.3 \pm 3.5$ & $29.6 \pm 14.7^{*}$ & $30.0 \pm 5.5^{*}$ & $26.0 \pm 3.4^{*}$ & WM \\
\hline
\end{tabular}

${ }^{a}$ Negative control: DMSO; Spontaneous revertant colonies: 18-20; Pozitif control: Mitomycin-C (0.5 $\mu \mathrm{g}$ plate $\left.{ }^{-1}\right)$ : No growth. *Analysis of mutagenic activity of sediments from Nif brook as the number of $\mathrm{His}^{+}$revertants in Ames test without metabolic activation system; Chi-square $\left(\alpha^{2}\right)$ test, significant data are shown in $\left({ }^{*}\right)(\mathrm{p}<0.005)$. A-Cr: Ames Criteria; SM: Strongly Mutagenic; M: Moderate mutagenic; WM: Weakly Mutagenic and NM: Non Mutagenic

Table 2: Mutagenicity analysis of sediment samples using $S$. typhimurium assay with TA 100 strain in the absence of metabolic activation

\begin{tabular}{llllll}
\hline TA 100 & $\mathrm{NC}^{\mathrm{a}}$ & $25 \mu \mathrm{g}$ & $50 \mu \mathrm{g}$ & $100 \mu \mathrm{g}$ & $\mathrm{A}-\mathrm{Cr}$ \\
\hline
\end{tabular}

$\begin{array}{llllll}\text { Nif } 1 & 121.0 \pm 17.0 & 144.0 \pm 31.00^{*} & 136.0 \pm 2.00^{*} & 99.0 \pm 1.00^{*} & \text { Toxic }\end{array}$

$\begin{array}{llllll}\text { Nif } 2 & 106.6 \pm 3.50 & 149.5 \pm 2.500^{*} & 119.0 \pm 9.00^{*} & 114.3 \pm 6.60 & \mathrm{NM}\end{array}$

$\begin{array}{llllll}\text { Nif } 3 & 106.6 \pm 3.50 & 126.0 \pm 9.500^{*} & 131.0 \pm 9.50^{*} & 135.0 \pm 11.5^{*} & \mathrm{NM}\end{array}$

Nif $4 \quad 106.6 \pm 3.50 \quad 95.0 \pm 15.10^{*} \quad 89.3 \pm 9.29^{*} \quad 63.3 \pm 5.80^{*} \quad$ Toxic

$\begin{array}{llllll}\text { Nif } 5 & 106.6 \pm 3.50 & 130.0 \pm 14.70^{*} & 117.5 \pm 5.50 & 122.0 \pm 3.40^{*} & \text { NM }\end{array}$

${ }^{a}$ Negative control: DMSO; Spontaneous revertant colonies: 107-117; Pozitif control: $\mathrm{NaN}_{3}\left(1.5 \mu \mathrm{g}\right.$ plate $\left.\mathrm{e}^{-1}\right)$ : 980-1600; *Analysis of mutagenic activity of sediments from Nif brook as the number of $\mathrm{His}^{+}$revertants in Ames test without metabolic activation system; Chi-square $\left(\chi^{2}\right)$ test, signific ant data are shown in $(*)(\mathrm{p}<0.005)$. A-Cr: Ames Criteria, SM: Strongly Mutagenic; M: Moderately mutagenic; WM: Weakly Mutagenic and NM: Non Mutagenic

their mutagenicity. The mutagenicity results for DMSO-dissolved test samples from sediment of both strains are shown in Table 1 and 2. The results in Table 1 and 2 were obtained according to the criteria for Ames test system. Figure 2 graphically shows the revertant colonies due to mutagenicity from each of the sites and strains.

For TA 98 strain, the greatest numbers of revertants were observed at Nif 5 (Number of revertant colonies were $30(\mathrm{p}<0.005)$, respectively) and weak mutagenicity was observed for the sediment sample. Other samples were not mutagenic.

For TA 100 strain, no mutagenicity was observed for the sediment samples at site 5 whereas the lowest numbers were detected at sites 1 and 4 on Nif brook. Apart from this, toxicity was detected in 2 of the sediment samples from Nif 1-4 for TA 100 strain.

In the screening assays using multiple concentrations, the sample was considered to be mutagenic when the number of revertant colonies in the test plates was double that of revertants in the solvent control (Maron and Ames, 1983). Mutagenicity of the samples as studied at different concentrations was evaluated according to the significance in the linear portion of the dose-response curve. Dose-related increase in the number of the revertants was considered as the evidence of mutagenicity (Mortelmans and Zeiger, 2000). Toxic effects of the sediment samples on bacteria were

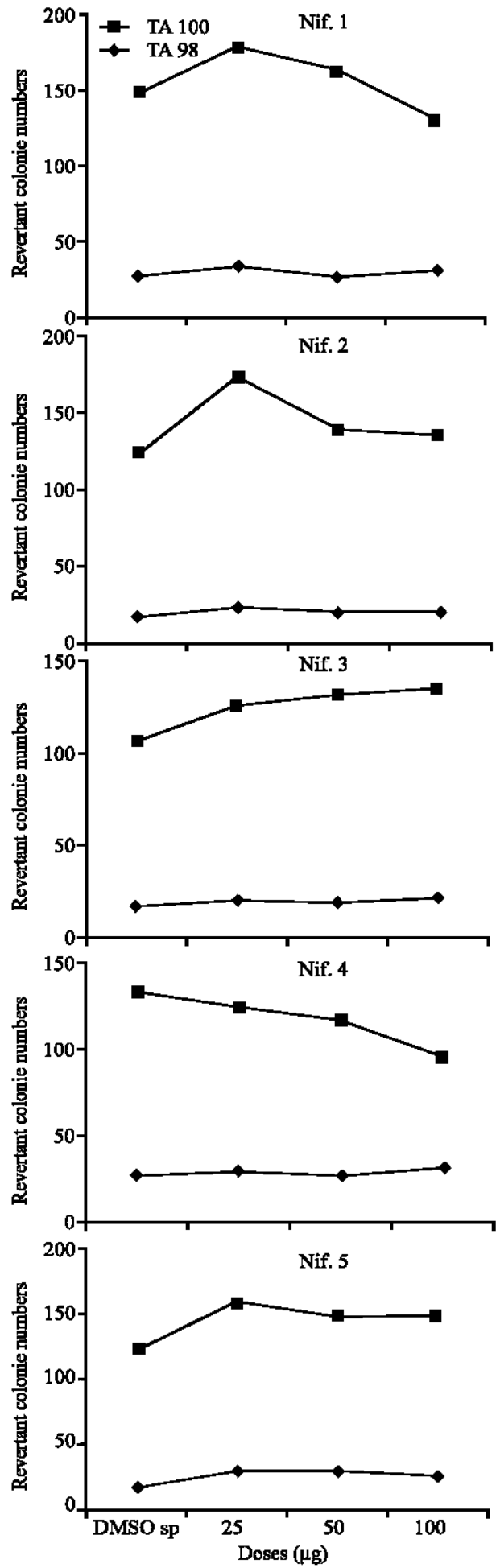

Fig. 2: Mutagenicity of the sediment samples from the Nif brook (NC: Values of solvent control (DMSO))

observed on the basis of significantly reduced numbers of revertants compared to the solvent controls 
(Kutlu et al., 2004). The present study aimed to assess the mutagenicity of sediment samples from Nif brook. According to Ames criteria, sediment samples from the Nif 5 were found to be mutagenic at TA 98 strain. The toxic effects of the sediment samples were observed on the samples from Nif 1 through 4 for TA 100 strain.

To the knowledge, the current study is the 2nd mutagenicity and genotoxicity assessment in Nif brook using the Ames test system. Boyacioglu et al. (2008) found mutagenicity on the same site with TA 98 strain in their mutagenicity study performed by Soxhlet extraction method on the samples taken from Nif brook in 2008. Arslan et al. (2009) observed developmental abnormalities in the embryos of sea urchins in the embryonic toxicity study they performed with sea urchins on water and sediment samples from the same site in 2009 and concluded that sediment and water samples of Nif brook were toxic (Arslan et al., 2009).

Similar to the results, mutagenicity and toxicity was found in sediment samples from Porsuk river (Kutlu et al., 2004). MN mutagenicity test was made in order to determine mutagenicity of sediment samples of Kars brook and information was obtained on mutagenicity of the sediment (Ozkan et al., 2009).

Complex interactions among chemicals confound the results of such studies and thereby, make their interpretation difficult. TA 98 strain of $S$. typhimurium is known to be more resistant to toxic materials than TA 100 strain (Abe and Urano, 1994). TA 98 detects frameshift mutagens (e.g., polycyclic hydrocarbons) and TA 100 for base-pair mutagens are widely used in general mutagenicity testing (Maron and Ames, 1983).

Mutagenicity of the samples as studied at different concentrations was evaluated according to the significance in the linear portion of the dose-response curve. Dose-dependent increase in the number of the revertants was considered as evidence of mutagenicity (Mortelmans and Zeiger, 2000). Toxic effects of the sediment samples on bacteria were observed on the basis of significantly reduced numbers of revertants compared to the solvent controls (Kutlu et al., 2004).

Organized Industrial Parks (OIPs) are considered to be one of the sources of this pollution because wastewater from Kemalpasa OIP is discharged into Nif brook causing significant pollution. No common treatment facility exists in Kemalpasa OIP as well as none of the settlements around Kemalpasa possesses a treatment facility. One of the most important pollution sources is leakage of water from wastes and hazardous materials into the bed of brook, especially in its part passing through the OIP.

Considering that the water from the branches of Nif brook is being used for irrigation and stock-raising, one can easily understand the extent of the hazards of pollution in Nif brook and therefore, the 1st step to eliminate this should be control of the polluting sources.

\section{CONCLUSION}

This study is second mutagenic investigating for Nif brook but it is used a different methods on the extractions of sediment samples. As a result, sediments samples from Nif brook were found to be mutagenic with solvent extraction method.

\section{ACKNOWLEDGEMENTS}

The present study was conducted in the contex of Scientific Research Project of Celal Bayar University, Faculty of Art and Science, Biology Department (Project No.: FEF 2006-058).

\section{REFERENCES}

Abe, A. and K. Urano, 1994. Influence of chemicals commonly found in a water environment on the Salmonella mutagenicity test. Sci. Total Environ., 153: $169-175$.

Alexander, M., 1981. Biodegradation of chemicals of environmental concern. Science, 211: 132-138.

Ames, B.N., J. McCann and E. Yamasaki, 1975. Methods for detecting carcinogens and mutagens with Salmonella/mammalian microsome mutagenicity test. Mut. Res., 31: 347-364.

Arslan, O.C., H. Parlak, S. Katalay, M. Boyacioglu and M.A. Karaslan, 2009. Embryotoxic effects of water and sediment from Nif Brook (Western, Turkey) on sea urchin Paracentrotus lividus. Fresenius Environ. Bull., 18: 663-664.

Boyacioglu, M., H. Parlak, R. Oral and O. Cakal, 2008. Mutagenicity of sediment and water samples from Nif Brook (Western Turkey). Fresenius Environ. Bull., 17: 9-15.

Czyz, A., H. Szpilewska, R. Dutkiewicz, W. Kowalska, A. Biniewska-Godlewska and G. Wegrzyn, 2002. Comparison of the Ames test and a newly developed assay for detection of mutagenic pollution of marine environments. Mutat. Res., 519: 67-74.

Furlong, E.T., D.S. Carter and R.A. Hites, 1988. Organic contaminants in sediments from the trenton channel of the Detroit River, Michigan. J. Great Lakes Res., 14: 489-501.

Kataoka, H., T. Hayatsu, G. Hietsch, H. Steinkellner and S. Nishioka et al., 2000. Identification of mutagenic heterocyclic amines(IQ.Trp-P-1 and A $\alpha$ C) in the water of the Danube River. Mutat. Res., 466: $27-35$. 
Kotelevtsev, S.V. and L.I. Stepanova, 1995. Biochemical and Genotoxicological Monitoring of Ecosystems with Special Reference to Lake Baikal and Northern Black Sea. In: Molecular Aspects of Oxidative Drug Metabolizing Enzymes: Their Significance in Environmental Toxicology, Chemical Carcinogenesis and Health, Arinc, E., J.B. Schenkman and E. Hodgson (Eds.). Springer-Verlag, Berlin, Heidelberg, pp: 567-589.

Kutlu, M., G. Aydogan, F. Susuz and A. Ozata, 2004. The Salmonella mutagenicity of water and sediments from the Porsuk River in Turkey. Environ. Toxicol. Pharmacol., 17: 111-116.

Maccubin, A.E., N. Ersing and M.E. Frank, 1991. Mutagenicity of sediments from the Detroit river. J. Great Lake Res., 17: 314-321.
Maron, D.M. and B.N. Ames, 1983. Revised methods for the Salmonella mutagenicity test. Mutat. Res., 113: 173-215.

Mortelmans, K. and E. Zeiger, 2000. The Ames Salmonella/microsome mutagenicity assay. Mutat. Res., 455: 29-60.

Ozkan, O., S. Gul, O. Keles, P. Aksu, T.O. Kaya and G. Nur, 2009. The investigation of the mutagenic activity of Kars River sediments on Orthrias angorae (Steindachner, 1897). Kafkas Univ. Vet. Fakultesi Dergisi, 15: 35-40.

Schuetzle, D. and J. Lewtas, 1986. Bioassay-directed chemical analysis in environmental research. Anal. Chem., 58: 1060-1075.

Shugart, L.R., 1995. Environmental Genotoxicology. In: Fundamentals of Aquatic Toxicology, Rand, G.M. (Ed.). Taylor and Francis Publishers, USA., pp: 405-419. 